Dhaka Univ. J. Biol. Sci. 19(2): 189-194, 2010 (July)

\title{
NOVEL FUNCTIONS OF NON-O1 NON-O139 VIBRIO CHOLERAE LIPOPOLYSACCHARIDE FOR ITS HAEMAGGLUTINATING ACTIVITY
}

\author{
Summaiya F. Khan ${ }^{1}$, Fazle Rabbi, Nazmul Ahsan ${ }^{1}$, Mahmuda Yasmin, \\ JAMALUn Nessa AND CHOWdHury R. AHSAN* \\ Department of Microbiology, University of Dhaka, Dhaka-1000, Bangladesh
}

Key words: Non-O1 non-O139 V. cholerae, LPS, Haemagglutination

\begin{abstract}
Haemagglutinating activity of the surface lipopolysaccharide (LPS) of nonO1 non-O139 Vibrio cholerae was assayed against rabbit and chicken erythrocytes. The serially diluted non-O1 non-O139 $\mathrm{V}$. cholerae LPS showed haemagglutination of rabbit erythrocytes up to a dilution of $1: 512$, which is probably due to the hydrophilic polysaccharide moiety of the LPS. To test whether the LPS was able to bind to glycoproteins and other simple sugars, the LPS was incubated with mucin, glucose and sucrose and was assayed for the residual haemagglutinating activity. It was found that the mucin could inactivate the LPS, which indicated that the LPS of the non-O1 non-O139 V. cholerae strains may bind to glycoprotein and its mechanism of adherence may be similar to that of other bacterial LPS. However, the glucose and sucrose could not inhibit the LPS as has been demonstrated by the positive residual haemagglutinating activity of the LPS.
\end{abstract}

\section{Introduction}

Vibrio cholerae $\mathrm{O} 1$ organisms are known to be the causative agents of the fifth, sixth and seventh pandemics of cholera. These organisms are biochemically indistinguishable from their non-O1 $\mathrm{V}$. cholerae counterparts except for their property to be agglutinated by O1 antiserum.(1) Non-O1 $V$. cholerae strains which represent a heterogeneous group comprising of more than 155 serogroups, are the natural inhabitants of aquatic environment and are known to be associated with prawns, shellfishes, etc.(2) Some of these strains are also reported to be responsible for sporadic cases and/or limited outbreaks of gastro-enteritis and extra-intestinal infections.(3) However, in 1992-1993, an epidemic of cholera was reported from India and Bangladesh which was caused by a non-O1 strain of $V$. cholerae, later to be designated as O139 Bengal.(4)

The other sero-group, non-O1 non-O139 V. cholerae comprise a heterogeneous group of organisms whose clinical association with humans is inadequately understood. However, it is now clear beyond doubt that some strains of non-O1 non-O139 V. cholerae have the capacity to cause a cholera-like syndrome and the capacity to flare into a localized outbreak..$^{(5)}$ Recently we reported that the environmental non-O1 non-O139 $\mathrm{V}$. cholerae strains produce toxins, other than cholera toxin.(6) Although the study clarified the role of different toxins, including heat-labile enterotoxin, haemolysin and/or

*Corresponding author. <crahsan@univdhaka.edu>. ${ }^{1}$ Department of Genetic Engineering and Biotechnology, University of Dhaka, Dhaka-1000, Bangladesh. 
cytotoxin, in the pathogenesis of the non-O1 non-O139 V. cholerae, however, the study did not characterize the role of lipopolysaccharides (LPS) in the virulence process of the bacteria.

High haemagglutinating activity was demonstrated by the LPS of the V. cholerae O139 strains.(7) The bacterial haemagglutinin causing agglutination of erythrocytes has been related to the bacterial adherence to the intestinal mucosa, because the erythrocyte membrane has been believed to possess homolog(s) of the mucosal substance(s) involved in adherence. ${ }^{(7,8)}$ In the present study, we provide data on the role of LPS in the haemagglutinating activity, which may be helpful in characterizing these virulence factors for a better understanding of the pathogenic mechanisms of the environmental non-O1 non-O139 V. cholerae strains.

\section{Materials and Methods}

Surface water was taken from small water reservoirs, ponds and rivers around Dhaka city. Water samples were enriched in alkaline peptone water at $37^{\circ} \mathrm{C}$ for overnight and were then inoculated on thiosulphate citrate bile salt sucrose (TCBS) agar plate. After overnight incubation for $18-24 \mathrm{hr}$, suspected yellow colonies were subcultured on gelatin agar (GA) and were identified by a series of biochemical tests. Serotyping was performed by slide agglutination with specific antiserum and only three strains were confirmed as non-O1 non-O139 V. cholerae, which were preserved in $\mathrm{T}_{1} \mathrm{~N}_{1}$ (1\% trypticase, $1 \% \mathrm{NaCl}$ ) soft agar at room temperature. The $V$. cholerae $569 \mathrm{~B}$, which is a toxigenic $\mathrm{O} 1$ vibrio strain, was collected from the stock culture of the Department of Microbiology, University of Dhaka and was used as a positive control.

LPS from both non-O1 non-O139 V. cholerae and $V$. cholerae 569B were extracted following the method of Westphal and Jann.(9) Bacteria were grown overnight in Brain Heart Infusion (BHI) broth and the cells were collected by centrifugation at 6,000 rpm for $10 \mathrm{~min}$ for LPS extraction. Approximately $5 \mathrm{gm}$ of the bacteria were suspended in $10 \mathrm{ml}$ of distilled water and were stirred with $10 \mathrm{ml}$ of $90 \%$ phenol vigorously for $10 \mathrm{~min}$ in a water bath at $68^{\circ} \mathrm{C}$. They were cooled to $10^{\circ} \mathrm{C}$ and centrifuged at 4,000 rpm for $30 \mathrm{~min}$. After centrifugation, the aqueous layer containing LPS was removed and dialyzed for 48 $\mathrm{hr}$ against distilled water with several changes. The LPS was then lyophilized and the dry LPS was dissolved in $20 \mathrm{mM}$ tris- $\mathrm{HCl}$ containing $0.9 \% \mathrm{NaCl}(\mathrm{pH} 7.5)$ to achieve a final concentration of $1 \mathrm{mg} / \mathrm{ml}$.

The haemagglutinating activity of the non-O1 non-O139 V. cholerae LPS $(1 \mathrm{mg} / \mathrm{ml})$ was assayed by the method of Jones et al. (10) A serial dilution of $50 \mu \mathrm{l}$ of the LPS samples were made in $20 \mathrm{mM}$ tris- $\mathrm{HCl}$ buffer containing $0.9 \% \mathrm{NaCl}(\mathrm{pH} 7.5)$ and was mixed with equal volume of $1.5 \%$ rabbit erythrocytes (made in the same buffer) in the wells of a 96 well polystyrene V-bottom microtiter plate. The plate was incubated at room temperature for $45 \mathrm{~min}$. The haemagglutinating activity unit was defined as the 
reciprocal of the highest dilution of the LPS sample causing visible agglutination of the erythrocytes. LPS of $V$. cholerae 569B, assayed in the same manner, was used as the positive control. The same process was also performed with chicken erythrocytes, to check the haemagglutinating activity of the LPS samples. Wells containing erythrocytes mixed with only buffer were used as negative controls.

The LPS preparations were hydrolyzed with $1 \%$ acetic acid at $100^{\circ} \mathrm{C}$ for $90 \mathrm{~min}$. The lipid A fraction was spun down by centrifuging the solution at 3,000 rpm for $10 \mathrm{~min}$. The supernatant containing the polysaccharide moiety was checked for haemagglutinating activity following the method described above.

Glycoprotein (mucin) and sugars (glucose and sucrose) $(1 \mathrm{mg} / \mathrm{ml})$ were serially diluted and incubated with the LPS $(1 \mathrm{mg} / \mathrm{ml})$ of non-O1 non-O139 V. cholerae at $37^{\circ} \mathrm{C}$ for $30 \mathrm{~min}$. Residual haemagglutinating activity of the LPS preparation against rabbit erythrocytes was determined following the method described above. Glycoprotein and sugars incubated with the LPS of $V$. cholerae 569B and checked for haemagglutinating activity, were served as positive controls.

\section{Results and Discussion}

In the progression of enteric infections, bacterial adherence to the surface of host intestinal mucosa has been established as an essential step. This step has been documented to be accomplished by the factors possessing the ability to agglutinate erythrocytes. The factors causing haemagglutination are well-known as haemagglutinins, which recognize the specific binding sites, the so called receptors. Since erythrocytes from different animal species possess different types of receptors, the haemagglutininerythrocyte interaction provides the nature of the receptor(s) involved in the mucosal receptions of pathogenic bacteria. For several intestinal pathogens, including $V$. cholerae, a correlation between bacterial haemagglutinating property and adhesiveness has been found. (11-13) In this study, the haemagglutinating activity of the surface LPS of the isolated non-O1 non-O139 strain of $V$. cholerae was assayed against rabbit and chicken erythrocytes (Fig. 1). The serially diluted non-O1 non-O139 V. cholerae LPS showed haemagglutination of rabbit erythrocytes up to a dilution of $1: 512$. The results indicated that the non-O1 non-O139 $\mathrm{V}$. cholerae strains had strong adhesive property and hence were pathogenic. Again as the non-O1 non-O139 V. cholerae LPS caused haemagglutination of rabbit erythrocytes, not chicken, therefore, it can be concluded that the non-O1 non-O139 $V$. cholerae LPS needs specific receptors for binding on the mucosal surfaces.

LPS is composed of polysaccharide moiety and the lipid A, which gives the hydrophobic nature and remains embedded in the outer leaflet of the outer membrane. To clarify whether the haemagglutinating activity of the LPS was due to the polysaccharide moiety, the LPS was subjected to acid hydrolysis using $1 \%$ acetic acid and was assayed for haemagglutinating activity. The result showed that haemagglutinating 
activity was associated with the hydrophilic polysaccharide moiety of the LPS. Considering the cell surface architecture, direct interaction of the outer membrane protein with the host mucosa seems to be impossible. In contrast, the extended polysaccharide moiety of LPS may be the first to come in contact with the mucosal membrane and from this study, it seems likely that the polysaccharide moiety with strong haemagglutinating activity may function as the ubiquitous adhesin in these enteropathogenic vibrios.

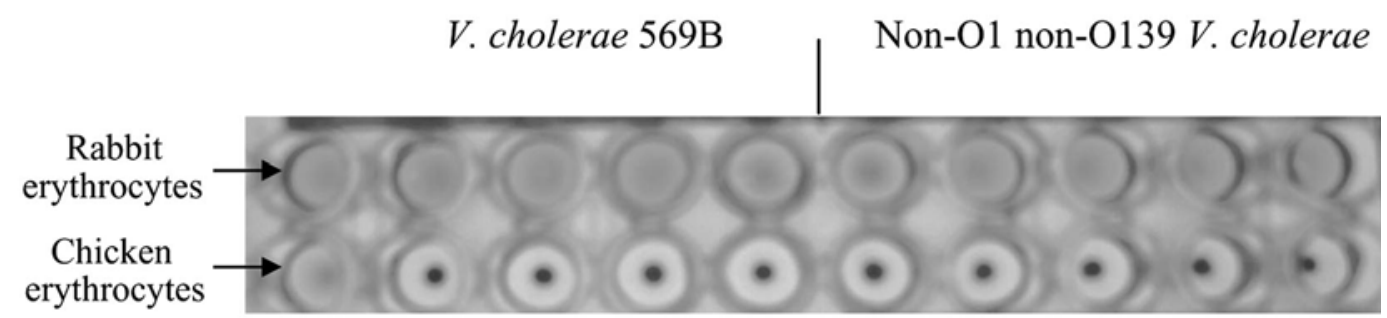

Fig. 1. Positive haemagglutination activity was demonstrated by non-O1 non-O139 V. cholerae and $V$. cholerae 569B for rabbit erythrocytes. The chicken erythrocytes, however, showed negative results.

The LPS binding protein in the human body is a glycoprotein with the ability to potentiate the bactericidal activity of human bactericidal or permeability-increasing protein through specific binding to LPS.(14) Although it is not known whether the homologs of the LPS binding protein is contained in the mucosa of human small intestine, the haemagglutinating activity and the affinity to glycoproteins may indicate that Gram-negative bacterial enteropathogens may employ LPS in the glycoprotein mediated anchorage to the host intestinal mucosa. ${ }^{(15)}$ To test whether the LPS was able to bind to glycoproteins, the LPS sample was incubated with a glycoprotein, mucin and was assayed for the residual haemagglutinating activity. It was found that the mucin could inactivate the LPS, which indicated that the LPS of the non-O1 non-O139 V. cholerae strains may bind to glycoprotein and its mechanism of adherence may be similar to that of other bacterial LPS. $(7,15)$ The LPS was also incubated with glucose and sucrose in a similar fashion. However, they could not inhibit the LPS as has been demonstrated by the positive residual haemagglutinating activity of the LPS. The inability of any sugar to abolish the adherence of $V$. cholerae $\mathrm{O} 1$ to the mucous coat of the human small intestine was documented by others. ${ }^{(16)}$ This suggests involvement of the non-sugar mucosal component(s) in the adherence of this human pathogen. Since the major part of the small intestine is covered with a thick mucous layer containing glycoproteins, ${ }^{(17)}$ sufficient elimination of the haemagglutinating ability of any vibrio LPS with the polypeptide portion of the glycoprotein may indicate the possibility of LPS-mediated intestinal adherence in the enteropathogenic vibrios, including the non-O1 non-O139 V. cholerae. 
However, further studies can be performed to identify the receptors with which the nonO1 non-O139 V. cholerae LPS interacts.

\section{References}

1. Shimada TE, E Arakawa, K Itoh, T Okitsu, A. Matsushima, Y Asai, S. Yamai, T Nakazato, GB Nair, MJ Albert and Y Takeda 1994. Extended serotyping scheme for Vibrio cholerae. Curr. Microbiol. 28: 175-178.

2. Nair GB, RK Bhadra, T Ramamurthy, A Ramesh and SC Pal 1991. Vibrio cholerae and other vibrios associated with paddy field cultured prawns. Food Micriobiol. 8: 203-8.

3. Blake PA, RW Weaver and DG Hollis 1980. Diseases of humans (other than cholera) caused by vibrios. Annual Rev. Microbiol. 34: 341-367.

4. Albert MJ, AK Siddique, MS Islam, AS Faruque, M Ansaruzzaman, SM Faruque and RB Sack 1993. Large outbreak of clinical cholera due to Vibrio cholerae non-O1 in Bangladesh. Lancet 341: 704.

5. Ramamurthy T, S Garg, R Sharma, SK Bhattacharya, GB Nair, T Shimada, T takeda, T. Karasawa, H Kurazano and A Pal 1993. Emergence of novel strain of Vibrio cholerae with epidemic potential in Southern and Eastern India. Lancet 341: 703-704.

6. Begum K, CR Ahsan, M Ansaruzzaman, DK Dutta, QS Ahmad and KA Talukder 2006. Toxin(s), other than cholera toxin, produced by environmental non-O1 non-O139 Vibrio cholerae. Cell. Mol. Immunol. 3: 115-121.

7. Alam M, S Miyoshi, K Tomochika and S Shinoda 1997. Haemagglutination is a novel biological function of lipopolysaccharide (LPS), as seen with the Vibrio cholerae O139 LPS. Clin. Diagn. Lab. Immunol. 64: 4035-4041.

8. Alam M, S Miyoshi, S Yamamoto, K Tomochika and S Shinoda 1996. Expression of virulence related properties by, and intestinal adhesiveness of Vibrio mimicus strains isolated from aquatic environments. Appl. Environ. Microbiol. 62: 3871-3874.

9. Westphal, O and K Jann 1965. Bacterial lipopolysaccharides: extraction with phenol-water and further applications of the procedure. Methods Carbohydr. Chem. 5: 83-91.

10. Jones GW, GD Abrams and R Freter 1976. Adhesive properties of Vibrio cholerae: Adhesion to isolated rabbit brush border membranes and haemagglutinating activity. Infect. Immun. 14: 232-239.

11. Nagayama K, T Oguchi, M Arita and T Honda 1994. Correlation between cell-associated mannose-sensitive haemagglutination by Vibrio parahemolyticus and adherence to a human colonic cell line Caco-2. FEMS Microbiol. Lett. 120: 207-210.

12. Nagayama K, T Oguchi, M Arita and T Honda 1995. Purification and characterization of a cellassociated haemagglutinin of Vibrio parahemolyticus. Infect. Immun. 63: 1987-1992.

13. Yamamoto $\mathrm{T}, \mathrm{T}$ Kamano, $\mathrm{M}$ Uchimura, M Iwanaga and T Yokota 1988. Vibrio cholerae O1 adherence to villi and lymphoid follicle epithelium: In vitro model using formalin treated human small intestine and correlation between adherence and cell-associated haemagglutination levels. Infect. Immun. 56: 3241-3250.

14. Horwitz AH, RE Williams and G Nowakowski 1995. Human lipopolysaccharide binding protein potentiates bactericidal activity of human bactericidal/permeability increasing protein. Infect. Immun. 63: 522-527. 
15. Alam M, S Miyoshi, S Yamamoto, K Tomochika and S Shinoda 1997. Vibrio mimicus attaches to the intestinal mucosa by outer membrane haemagglutinins specific to the polypeptide moieties of glycoproteins. Infect. Immun. 65: 3662-3665.

16. Yamamoto $\mathrm{T}$ and $\mathrm{T}$ Yokota 1988. Electron microscopic study of Vibrio cholerae $\mathrm{O} 1$ adherence to the mucus coat and villus surface in the human small intestine. Infect. Immun. 56: 27532759.

17. Uchimura M and T Yamamoto 1992. Production of hemagglutinins and pilli by Vibrio mimicus and its adherence to human and rabbit small intestines in vitro. FEMS Microbiol. Lett. 91: 73-78.

(Manuscript received on 25 August, 2009; revised on 30 March, 2010) 